\title{
Multiobjective Optimization of 6-DOF Parallel Manipulator for Desired Total Orientation Workspace
}

\author{
Hongbin Qiang $\mathbb{D}$, ${ }^{1}$ Lihang Wang, ${ }^{1}$ Jisong Ding, ${ }^{1}$ and Lijie Zhang $\mathbb{D}^{1,2}$ \\ ${ }^{1}$ Hebei Key Laboratory of Heavy Machinery Fluid Power Transmission and Control, Yanshan University, \\ Qinhuangdao 0660004, China \\ ${ }^{2}$ Key Laboratory of Advanced Forging \& Stamping Technology and Science, Yanshan University, Qinhuangdao 066004, China
}

Correspondence should be addressed to Lijie Zhang; ljzhang@ysu.edu.cn

Received 2 January 2019; Revised 27 March 2019; Accepted 7 April 2019; Published 22 April 2019

Academic Editor: Javier Moreno-Valenzuela

Copyright (c) 2019 Hongbin Qiang et al. This is an open access article distributed under the Creative Commons Attribution License, which permits unrestricted use, distribution, and reproduction in any medium, provided the original work is properly cited.

\begin{abstract}
A desired total orientation workspace for a parallel manipulator is usually an essential requirement in a practical application. At present, for the multiobjective optimization method of 6-DOF parallel manipulator for desired total orientation workspace, it is needed to predefine maximal and minimal lengths of the legs to serve as the constraint, and then the numerical method is used to solve the length of the legs and judge whether the solved maximal and minimal leg lengths meet the constraint. Predefining maximal and minimal length of the legs limits of the optimal range, the numerical method has heavy calculation burden and low calculating accuracy. In this paper, a hybrid method for solving the maximal and minimal lengths of the legs of 6-DOF parallel manipulator with desired total orientation workspace is proposed, and the actuator stroke length is calculated according to the maximal and minimal leg lengths. By judging whether the actuator stroke length can be solved to serve as the constraint, without the predefined maximal and minimal leg lengths to serve as the constraint, the optimal range is enlarged. Aiming at the physical size of the parallel manipulator and the proposed desired workspace condition index (DWCI), the optimization of the geometric parameters of the parallel manipulator is conducted based on the multiobjective optimization algorithm (NSGA-II), which is subject to the actuator stroke length. Stewart platform is set as the example; the geometric parameters of the platform whose workspace contains the desired total orientation workspace are optimized and the hybrid method is proved to be more accurate and efficient compared to the traditional numerical method. This method provides the optimization guidance for engineering designers to design the parallel manipulator for desired total orientation workspace.
\end{abstract}

\section{Introduction}

Parallel manipulator has the advantages of compact structure, strong bearing capacity, high precision of motion, and low inertia and therefore is widely used in the flight simulator, ship motion simulation, ship stabilized platform, space docking manipulator, parallel machine tools, and robot wrist $[1,2]$. However, the limited workspace and dexterity are the major defects of the parallel manipulator [3-6]. As a result, it is of great importance to design a set of geometric parameters of the parallel manipulator with ideal workspace and good performance.

At present, in the design of the parallel manipulator for the workspace requirements, there are two types [6]. One is to get the geometry parameters of the parallel manipulator that maximizes the workspace. Lou [6] defined the effective regular workspace and aimed at the maximal side length of the effective regular workspace for the constant orientation, and the optimization of the geometric parameters is conducted by the controlled random search (CRS) method, which is subject to the dexterity and the leg lengths. Toz [7] defined the dexterous workspace by the condition index and minimum singular value (MSV) and used the particle swarm optimization (PSO) to optimize the dexterous workspace of asymmetric parallel manipulator, which is subject to kinematics and geometric constraints. Aiming at the condition number, stiffness, and the reachable workspace solved by geometric method, Shirazi [8] optimized the geometric parameters of the 6-DOF parallel manipulator.

However, maximizing the workspace blindly does not meet the engineering requirements, since a desired regular shape workspace is usually an essential requirement in 


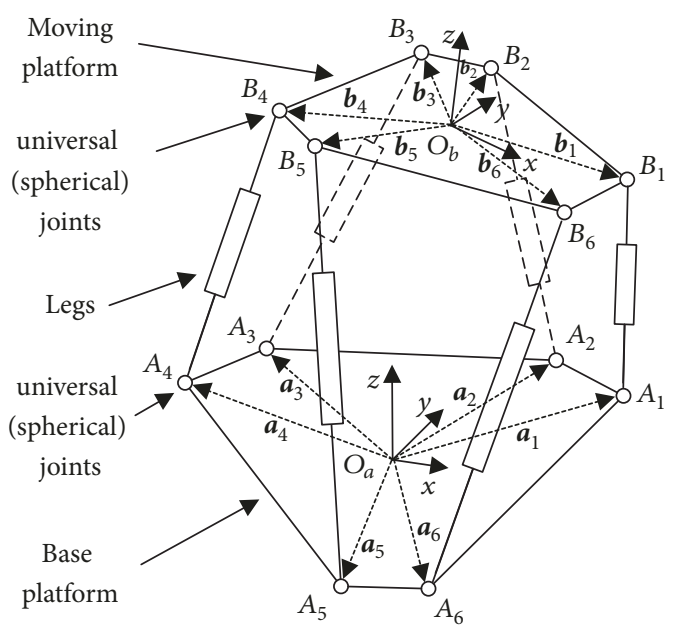

FIGURE 1: The structure sketch of 6-DOF parallel manipulator.

practice. The other type is to obtain the geometric parameters of a parallel manipulator whose workspace contains a desired workspace. Liu [9] used analytic method to design geometric parameters of a DELTA manipulator to get a desired total orientation workspace. In [10], a genetic algorithm was applied to optimize geometric parameters of a DELTA manipulator whose workspace contains a desired threedimensional workspace. As for a 6-DOF parallel manipulator whose workspace is six- dimensional, it is necessary to optimize geometric parameters of parallel manipulator for six- dimensional workspace. At present, some scholars have optimized the geometric parameters of the 6-DOF parallel manipulator for a desired lower-mobility workspace. Liu [11] optimized the geometric parameters of the 6-DOF parallel manipulator for a desired single DOF workspace. Cirillo [12] used genetic algorithm to optimize the geometric parameters of the 6-DOF parallel manipulator in aeronautics applications for the known trajectory. In addition, some scholars have designed geometric parameters of parallel manipulator for a desired total orientation workspace [13]. Zhao [14] exploited an algebraic algorithm to optimize the length of the legs of a parallel manipulator to get a desired total orientation workspace. $\mathrm{Fu}$ [15] proposed a novel 3-leg 6-DOF parallel manipulator whose three translational and three rotational motions are decoupled, and an algorithm of optimal designing is proposed to find the smallest dimensional parameters of the proposed robot for the desired workspace based on analytical solutions of the forward kinematics. Yang [16] gave the size and position of the desired total orientation workspace, and the leg lengths were obtained by the sixdimensional discretization of the workspace based on the numerical method, and then aiming at the linear combination of the physical size and dexterity index, the geometric parameters of the 6-DOF platform were optimized by the genetic algorithm, which is subject to the given leg lengths. Xie [17] solved the global transmission indexes for the desired total orientation workspace with different sets of geometric parameters; the optimal geometric parameters were obtained based on the changing characteristics of indexes with structural parameters.
At present, there are few studies on multiobjective optimization of 6-DOF parallel manipulator for desired total orientation workspace. In general, numerical method is used to discretize the six- dimensional desired total orientation workspace to solve the length of the legs; however, it consumes a lot of time. Furthermore, the solved length of the legs should be subject to the predefined maximal and minimal length, which limits the optimization design of the geometric parameters. Therefore, a hybrid method for solving the maximal and minimal length of the legs of 6-DOF parallel manipulator with desired total orientation workspace is proposed in this paper, and it is more accurate and efficient compared to the numerical method. And the actuator stroke length is calculated according to the maximal and minimal leg lengths, by judging whether the actuator stroke length can be solved to serve as the constraint; without the predefined maximal and minimal leg lengths to serve as the constraint, the optimal range is enlarged. And then aiming at the physical size of the parallel manipulator and the proposed DWCI, the optimization of the geometric parameters of the parallel manipulator is conducted based on the multiobjective optimization algorithm (NSGA-II), which is subject to the actuator stroke length. Stewart platform is set as the example; the geometric parameters of the platform whose workspace contains the desired total orientation workspace are optimized and the hybrid method is proved to be more accurate and efficient compared to the traditional numerical method. This method provides the optimization guidance for engineering designers to design the parallel manipulator for desired total orientation workspace.

\section{6-DOF Parallel Manipulator}

The structural sketch of 6-DOF parallel manipulator is shown in Figure 1. The manipulator is composed of the base platform, the moving platform, the legs, and the universal (spherical) joints. The basic platform is fixed and the moving platform is at the end of the manipulator. Two platforms are connected by the legs and the universal (spherical) joints.

Letting $A_{i}(i=1, \ldots, 6)$ denote the center points of the universal (spherical) joints which connect the legs and base platform and $B_{i}(i=1, \ldots, 6)$ denote the center points of the universal (spherical) joints which connect the legs and moving platform, $A_{i}$ and $B_{i}$ are at both ends of the same leg. A symmetrical hexagon is constructed by the point $A_{i}$, and $O_{a}$ is the center of the symmetrical hexagon, the point $O_{a}$ is the origin of the reference coordinate frame $\left\{O_{a}\right\}$, and the direction of $z$ axes perpendicular to the base platform points upwards, the direction of $x$ axes parallel to the base platform points to the midpoint of $A_{1}$ and $A_{6}$, and the direction of $y$ axes is determined by the right-hand rule. A symmetrical hexagon is constructed by the point $B_{i}$, and $O_{b}$ is the center of the symmetrical hexagon, the point $O_{b}$ is the origin of the reference coordinate frame $\left\{O_{b}\right\}$, and the direction of $z$ axes perpendicular to the moving platform points upwards, the direction of $x$ axes parallel to the moving platform points to the midpoint of $B_{1}$ and $B_{6}$, and the direction of $y$ axes is determined by the right-hand rule. The reference coordinate frame $\left\{\mathrm{O}_{a}\right\}$ and the moving coordinate frame $\left\{\mathrm{O}_{b}\right\}$ 
are assigned to the two platforms, respectively. The point $O_{a}$ is the origin of $\left\{\mathrm{O}_{a}\right\}$ located at the base platform and the point $O_{b}$ is the origin of $\left\{O_{b}\right\}$ located at the moving platform. The vectors $\boldsymbol{a}_{i}=\left[x_{a i}, y_{a i}, 0\right]$ and $\boldsymbol{b}_{i}=\left[x_{b i}, y_{b i}, 0\right]$ represent the vectors of the points $A_{i}$ and $B_{i}$ in $\left\{O_{a}\right\}$ and $\left\{O_{b}\right\}$, respectively. In the initial state, the $z$ axis of $\left\{O_{a}\right\}$ is recombined with the $z$ axis of $\left\{O_{b}\right\}$, the $x$ axis and $y$ axis of $\left\{O_{a}\right\}$ are parallel to the $x$ axis and $y$ axis of $\left\{O_{b}\right\}$, and the vector of the point $O_{b}$ in $\left\{\mathrm{O}_{a}\right\}$ is $\boldsymbol{h}=[0,0, H]$. The geometric parameters of the parallel manipulator can be determined by the vectors $\boldsymbol{a}_{i}, \boldsymbol{b}_{i}$, and $\boldsymbol{h}$.

2.1. Inverse Kinematics. The total orientation workspace of the 6-DOF parallel manipulator can be denoted as $\Omega=$ $\{(x, y, z, \alpha, \beta, \gamma)\}$, where the position in the workspace of the manipulator translation is defined as $\mathrm{P}=\{(x, y, z)\}$ and the posture in the workspace of the manipulator orientation is defined as $\mathrm{Q}=\{(\alpha, \beta, \gamma)\}$. In this paper, the RollPitch-Yaw (RPY) angles are used to describe the orientation transformation. First rotate coordinate frame $\left\{O_{b}\right\}$ around the $x$ axis of an angle $\gamma$ (Yaw), then rotate the resulting coordinate frame around the $y$ axis of an angle $\beta$ (Pitch), and finally rotate coordinate frame around the $z$ axis of an angle $\alpha$ (Roll). The transformation matrix from moving coordinate frame to the reference coordinate frame can be expressed as follows:

$$
\begin{aligned}
{ }_{B}^{A} \boldsymbol{R} & =\boldsymbol{R}(z, \alpha) \boldsymbol{R}(y, \beta) \boldsymbol{R}(x, \gamma) \\
& =\left[\begin{array}{ccc}
c \alpha c \beta & c \alpha s \beta s \gamma-s \alpha c \gamma & c \alpha s \beta c \gamma+s \alpha s \gamma \\
s \alpha c \beta & s \alpha s \beta s \gamma+c \alpha c \gamma & s \alpha s \beta c \gamma-c \alpha s \gamma \\
-s \beta & c \beta s \gamma & c \beta c \gamma
\end{array}\right]
\end{aligned}
$$

where $c \alpha \equiv \cos \alpha, s \alpha \equiv \sin \alpha, c \beta \equiv \cos \beta, s \beta \equiv \sin \beta, c \gamma \equiv$ $\cos \gamma$, and $s \gamma \equiv \sin \gamma$.

According to the position $\mathrm{P}$ and posture $\mathrm{Q}$ in the workspace and the transformation matrix ${ }_{B}^{A} R$, the leg length of parallel manipulator can be solved. The $i$ th leg vector can be obtained by the difference between the vectors $\boldsymbol{b}_{i}$ and $\boldsymbol{a}_{i}$ in $\left\{\mathrm{O}_{a}\right\}$. The $i$ th actuator can be expressed by (2). The vector diagram for the $i$ th actuator is given in Figure 2. $\left\{O_{b}\right\}$ represented by the dotted line is the initial state, and represented by the solid line is the arbitrary position state in the total orientation workspace.

$$
\begin{aligned}
\boldsymbol{l}_{i}= & \left({ }_{B}^{A} \boldsymbol{R} \cdot \boldsymbol{b}_{i}+{ }^{A} \boldsymbol{p}_{B}+\boldsymbol{h}\right)-\boldsymbol{a}_{i}=\left[\begin{array}{lll}
l_{i x} & l_{i y} & l_{i z}
\end{array}\right]^{\mathrm{T}} \\
= & {\left[\begin{array}{c}
c \alpha c \beta x_{b i}+(c \alpha s \beta s \gamma-s \alpha c \gamma) y_{b i}+x-x_{a i} \\
s \alpha c \beta x_{b i}+(s \alpha s \beta s \gamma+c \alpha c \gamma) y_{b i}+y-y_{a i} \\
-s \beta x_{b i}+c \beta s \gamma y_{b i}+z+H
\end{array}\right] }
\end{aligned}
$$

where ${ }^{A} P_{B}$ denotes the position $\mathrm{P}$.

Then the length of the $i$ th leg can be obtained by (3) and the square of the length of the $i$ th leg can be got by (4).

$$
\begin{aligned}
& e_{i}(x, y, z, \alpha, \beta, \gamma)=\sqrt{l_{i x}{ }^{2}+l_{i y}{ }^{2}+l_{i z}{ }^{2}} \\
& f_{i}(x, y, z, \alpha, \beta, \gamma)=l_{i x}{ }^{2}+l_{i y}{ }^{2}+l_{i z}{ }^{2}
\end{aligned}
$$

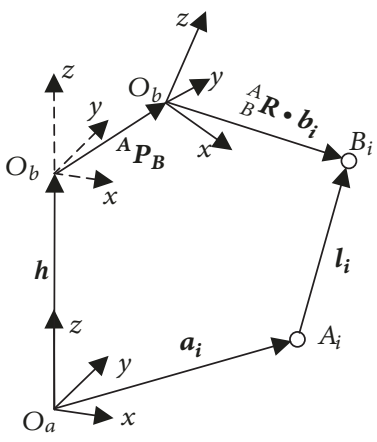

FIGURE 2: The vector diagram for the ith actuator.

\section{The Maximal and Minimal Leg Lengths and the Actuator Stroke Length}

The most usual types of workspace of 6-DOF parallel manipulator are reachable workspace, constant orientation workspace, orientation workspace, total orientation workspace, etc. Total orientation workspace is all the locations that may be reached with all the orientations among a set defined by ranges on the orientation angles [13]. The desired total orientation workspace is set by engineering designers according to engineering requirements. The desired total orientation workspace is a subset of the total orientation workspace, and it is usually geometry in a regular shape, such as a cube, a ball, and a cylinder. In this paper, a cube is set as the desired total orientation workspace $\Omega_{\mathrm{t}}$, as follows:

$$
\left.\begin{array}{l}
\Omega_{\mathrm{t}}=\{(x, y, z, \alpha, \beta, \gamma) \mid \\
x_{\min } \leq x \leq x_{\max }, y_{\min } \leq y \leq y_{\max } \\
z_{\min } \leq z \leq z_{\max }, \alpha_{\min } \leq \alpha \leq \alpha_{\max } \\
\beta_{\min } \leq \beta \leq \beta_{\max }, \gamma_{\min } \leq \gamma \leq \gamma_{\max }
\end{array}\right\}
$$

3.1. Numerical Method for Calculating the Maximal and Minimal Leg Lengths. Numerical method [18] involves discretizing the six-dimensional desired total orientation workspace, calculating the leg lengths for each pose (discretized positions and postures), and getting the maximal and minimal leg lengths. Specific steps are as follows.

(1) Set a set of geometric parameters of the 6-DOF parallel manipulator, which includes $\boldsymbol{a}_{i}, \boldsymbol{b}_{i}$, and $\boldsymbol{h}$.

(2) Divide the desired total orientation workspace $\Omega_{t}$ into the position workspace $\Omega_{\mathrm{p}}$ and the posture workspace $\Omega_{\mathrm{q}}$. The position workspace and the posture workspace can be expressed by (5) and (6), respectively, as follows:

$$
\begin{aligned}
& \Omega_{\mathrm{p}} \\
& =\left\{(x, y, z) \mid \begin{array}{c}
x_{\min } \leq x \leq x_{\max }, y_{\min } \leq y \leq y_{\max } \\
z_{\min } \leq z \leq z_{\max }
\end{array}\right\}
\end{aligned}
$$




$$
\left.\begin{array}{l}
\Omega_{\mathrm{q}} \\
=\left\{(\alpha, \beta, \gamma) \mid \begin{array}{c}
\alpha_{\min } \leq \alpha \leq \alpha_{\max }, \beta_{\min } \leq \beta \leq \beta_{\max } \\
\gamma_{\min } \leq \gamma \leq \gamma_{\max }
\end{array}\right.
\end{array}\right\}
$$

(3) Discretize the position and posture workspace by the grid method. The translation step lengths along $x$ axis, $y$ axis, and $z$ axis are $S_{x}, S_{y}$, and $S_{z}$. The orientation step lengths around $x$ axis, $y$ axis, and $z$ axis are $S_{\alpha}, S_{\beta}$, and $S_{\gamma}$. The number of discrete points in each dimension can be obtained by (8) as follows:

$$
N_{v}=\text { floor }\left(\frac{v_{\max }-v_{\min }}{S_{v}}\right)+1
$$

where floor $(\boldsymbol{\bullet})$ denotes a function for rounding down and the subscript $\mathrm{v}$ denotes each dimension $(v=x, y, z, \alpha, \beta, \gamma)$. Then, the total number of discrete points in all dimensions can be obtained by (9). The discretized position and posture can be expressed by (10) and (11).

$$
\begin{aligned}
& N_{T}=N_{x} N_{y} N_{z} N_{\alpha} N_{\beta} N_{\gamma} \\
& \quad\left[x_{m_{1}}, y_{m_{2}}, z_{m_{3}}\right] \\
& \quad=\left[x_{\min }+m_{1} S_{x}, y_{\min }+m_{2} S_{y}, z_{\min }+m_{3} S_{z}\right] \\
& {\left[\alpha_{n_{1}}, \beta_{n_{2}}, \gamma_{n_{3}}\right]} \\
& \quad=\left[\alpha_{\min }+n_{1} S_{\alpha}, \beta_{\text {min }}+n_{2} S_{\beta}, \gamma_{\text {min }}+n_{3} S_{\gamma}\right]
\end{aligned}
$$

where $m_{1}=0,1, \ldots, N_{x} ; m_{2}=0,1, \ldots, N_{y} ; m_{3}=$ $0,1, \ldots, N_{z} ; n_{1}=0,1, \ldots, N_{\alpha} ; n_{2}=0,1, \ldots, N_{\beta} ; n_{3}=$ $0,1, \ldots, N_{\gamma}$.

(4) According to (3), calculate the six leg lengths for each pose.

(5) Get the six maximal and minimal leg lengths by comparing the leg lengths in each position.

3.2. Algebraic Method for Calculating the Maximal and Minimal Leg Lengths. Equation (3) used to calculate the leg lengths is a six-dimensional function that involves the variables of $x, y, z, \alpha, \beta, \gamma$. Therefore, the maximal and minimal leg lengths are the maximum and minimum of a sixdimensional function. The functional maximum and minimum exist in the functional extremum or the boundary value. The extremal points of (3) are identical to (4) [19]. Therefore, for the convenience of solving the extremal points, we can obtain the extremal points of (3) by solving the extremal points of (4). Equation (12) is the partial derived function of (4), and the extremal points of (4) are obtained by (12) [20].

$$
\begin{aligned}
& \frac{\partial f}{\partial x}=2\left(c \alpha c \beta x_{b i}+(c \alpha s \beta s \gamma-s \beta c \gamma) y_{b i}+x-x_{a i}\right) \\
& \frac{\partial f}{\partial y}=2\left(s \alpha c \beta x_{b i}+(s \alpha s \beta s \gamma+c \alpha c \gamma) y_{b i}+y-y_{a i}\right) \\
& \frac{\partial f}{\partial z}=2\left(-s \beta x_{b i}+c \beta s \gamma y_{b i}+(z+H)\right)
\end{aligned}
$$

$$
\begin{aligned}
& \frac{\partial f}{\partial \alpha}=2\left(\left(x_{a i}-x\right)\left(s \alpha c \beta x_{b i}+\left(s \alpha s \beta s \gamma y_{b i}+c \alpha c \gamma\right) y_{b i}\right)\right. \\
& +\left(y-y_{a i}\right)\left(c \alpha c \beta x_{b i}+\left(c \alpha s \beta s \gamma y_{b i}-s \alpha c \gamma\right) y_{b i}\right) \\
& \frac{\partial f}{\partial \beta}=2\left(\left(\left(x_{a i}-x\right) c \alpha+\left(y-y_{a i}\right) s \alpha\right)\right. \\
& \left.\cdot\left(c \beta s \gamma y_{b i}-s \beta x_{b i}\right)-\left(c \beta x_{b i}+s \beta s \gamma y_{b i}\right)(z+H)\right) \\
& \frac{\partial f}{\partial \gamma}=2\left(\left(x-x_{a i}\right)(c \alpha s \beta c \gamma+s \alpha s \gamma)+\left(y-y_{b i}\right)\right. \\
& \cdot(s \alpha s \beta c \gamma-c \alpha s \gamma)+(z+H) c \beta c \gamma) y_{b i}
\end{aligned}
$$

The maximum and minimum of six-dimensional function exist in the extremum of six-dimensional function or the maximum and minimum of five-dimensional function (one of the six variables is the boundary point); the maximum and minimum of a five-dimensional function exist in the extremum of five-dimensional function or the maximum and minimum of four-dimensional function (two of the six variables are the boundary points). By this analogy, the rest can be done as shown in Figure 3. Particularly, the maximum and minimum of a one-dimensional function exist in the extremum of one-dimensional function or the maximum and minimum of a zero-dimensional function (all of the six variables equal to the boundary points). Since each variable has two boundary values and arbitrary combinations with other variables, the number of the functions of $\mathrm{k}$ variables can be obtained by

$$
N_{m}=2^{6-m} \times C_{6}^{6-m} \quad(m=1,2, \cdots, 6)
$$

where $m$ is the number of variables of the function.

The solving process of function of $\mathrm{k}$ variables is shown in Figure 3. The algebraic method contains solving the function of $m(m=1, \ldots, 6)$ variables, getting the extremal points of $m(m=1, \ldots, 6)$ variables and 64 boundary points, excluding the extremal points that are not in the desired total orientation workspace, and calculating the extremum of (3) by the obtained extremal points and boundary points. Specific steps are shown as follows.

(1) Set a set of geometric parameters of the 6-DOF parallel manipulator, which includes $\boldsymbol{a}_{i}, \boldsymbol{b}_{i}$, and $\boldsymbol{h}$.

(2) According to (12) and Figure 3, the function of $m(m=$ $1, \ldots, 6)$ variables is solved.

(3) Get the extremal points of $m(m=1, \ldots, 6)$ variables and 64 boundary points.

(4) Exclude the extremal points that are not in the desired total orientation workspace.

(5) Get the extremum and the boundary value of (3).

(6) Get the six maximal and minimal leg lengths by comparing the leg lengths obtained in step (5).

\subsection{Hybrid Method for Calculating the Maximal and Minimal} Leg Lengths. The numerical method takes much time to calculate the maximal and minimal leg lengths, due to the large calculation quantity on the leg length for each discretized 


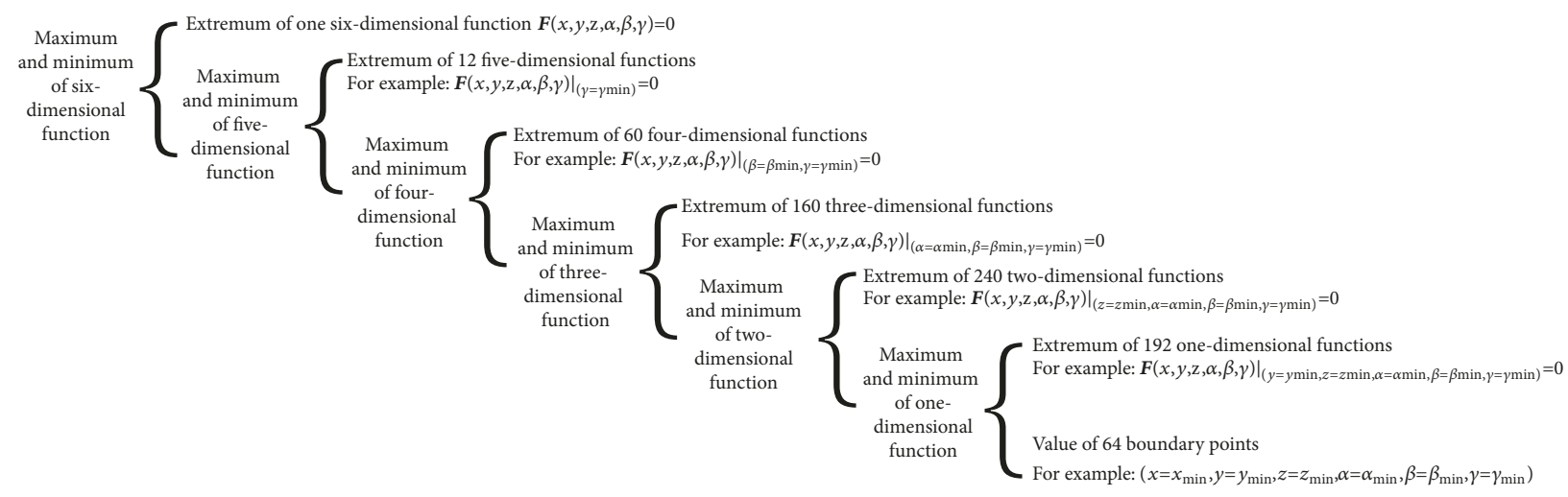

FIGURE 3: Solving process of function of $\mathrm{m}(\mathrm{m}=1, \ldots, 6)$ variables.

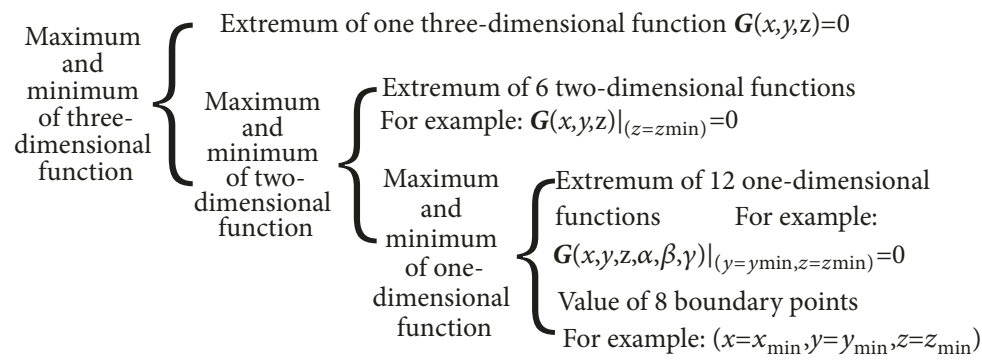

FIGURE 4: Solving process of function of $n(n=1,2,3)$ variables.

pose. And the precision is affected by the step lengths; usually a smaller step length leads to the higher precision and more time. The algebraic method takes less time to calculate the explicit maximal and minimal leg lengths. However, it is difficult to obtain the extremal points of the six-element function, and the extremal points of the function can be only obtained with the specified orientation variables $(\alpha, \beta, \gamma)$ which are equal to the boundary points.

Therefore, a hybrid method including the numerical method and algebraic method is proposed, where the numerical method is used for orientation variables and the algebraic method is used for translation variables. Firstly, the threedimensional posture workspace is discretized and the discretized postures are obtained. Then the functions of 3 variables $(x, y, z)$ in every discretized posture are solved. Finally, the maximal and minimal leg lengths are obtained. The extremal points can be obtained by the hybrid method based on the three-element function without solving six-element function. And compared with the numerical method, it is more accurate and efficient.

Equation (14) is the partial derived function of the functions of 3 variables $(x, y, z)$ and $(15)$ is the extremal points.

$$
\begin{aligned}
& \frac{\partial f}{\partial x}=c \alpha c \beta x_{b i}+(c \alpha s \beta s \gamma-s \beta c \gamma) y_{b i}+x-x_{a i} \\
& \frac{\partial f}{\partial y}=s \alpha c \beta x_{b i}+(s \alpha s \beta s \gamma+c \alpha c \gamma) y_{b i}+y-y_{a i} \\
& \frac{\partial f}{\partial z}=-s \beta x_{b i}+c \beta s \gamma y_{b i}+z+H
\end{aligned}
$$

$$
\begin{aligned}
& x=x_{a i}-c \alpha c \beta x_{b i}-(c \alpha s \beta s \gamma-s \beta c \gamma) y_{b i} \\
& y=y_{a i}-s \alpha c \beta x_{b i}-(s \alpha s \beta s \gamma+c \alpha c \gamma) y_{b i} \\
& z=s \beta x_{b i}-c \beta s \gamma y_{b i}-H
\end{aligned}
$$

The solving process of functions of $n(n=1,2,3)$ variables is shown in Figure 4. Specific steps of the hybrid method for solving the maximal and minimal leg lengths of 6-DOF parallel manipulator for desired total orientation workspace are shown as follows.

(1) Set a set of geometric parameters of the 6-DOF parallel manipulator, which includes $\boldsymbol{a}_{i}, \boldsymbol{b}_{i}$, and $\boldsymbol{h}$.

(2) Discretize the posture workspace by the grid method. The orientation step lengths around $x$ axis, $y$ axis, and $z$ axis are $S_{\alpha}, S_{\beta}, S_{\gamma}$. The discretized postures can be got by (11). The number of discrete points in each dimension can be got by (8). The total number of discretized postures can be got by (16).

$$
N_{T P}=N_{\alpha} N_{\beta} N_{\gamma}
$$

(3) Select one posture in $N_{T P}$ discretized postures.

(4) According to (14) and Figure 4, solve the function of $n(n=1,2,3)$ variables.

(5) Get the extremal points of $n(n=1,2,3)$ variables and 8 boundary points.

(6) Exclude the extremal points that are not in the desired total orientation workspace.

(7) Get the extremum and the boundary value of (3).

(8) Get the maximal and minimal leg lengths of each discretized posture. 


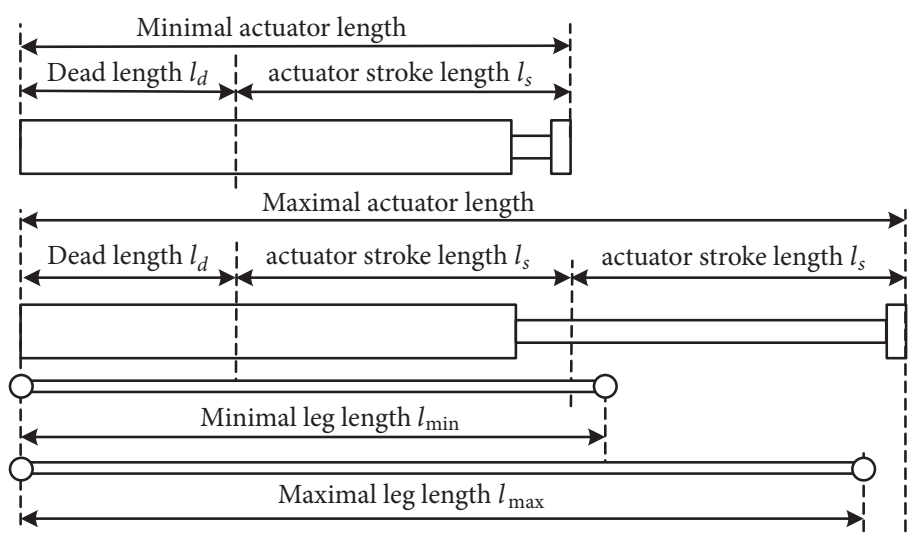

FIGURE 5: The schematic diagram of actuator.

(9) Select the next discretized posture in step (3) until all of the $N_{T P}$ discretized postures have been calculated.

(10) Get the six maximal and minimal leg lengths by comparing the leg lengths in the $N_{T P}$ discretized postures.

3.4. Actuator Stroke Length. The maximal and minimal leg length solved for the desired total orientation workspace should be between the maximum and minimum of the actuator length. The maximal and minimal values of the actuator can be determined by dead length and stroke length of the actuator. The dead length is the part which is not in the extension part of the cylinder, and the stroke length is the part in the extension part of the cylinder [21], as shown in Figure 5. According to Figure 5, the actuator stroke length can be obtained, as shown in (17).

$$
\begin{aligned}
& l_{s \min } \geq l_{\max }-l_{\min } \\
& l_{s \min } \geq \frac{\left(l_{\max }-l_{d}\right)}{2} \\
& l_{s \max } \leq l_{\min }-l_{d}
\end{aligned}
$$

where $l_{\min }$ denotes the minimum of leg length, $l_{\max }$ denotes the maximum of leg length, $l_{d}$ denotes the dead length of actuator, and $l_{s}$ denotes the actuator stroke length. Hydraulic cylinders, pneumatic cylinders, or electric cylinders are generally used as actuators, and the dead length $l_{d}$ usually can be determined according to the actual condition.

According to (17), the minimum $l_{s \text { min }}$ and the maximum $l_{\text {smax }}$ of the actuator stroke length can be got by (18) and (19), respectively. Equation (20) can be defined based on (18) and (19), which is expressed as follows:

$$
\begin{aligned}
l_{s \min } & =\max \left(\left(l_{\max }-l_{\min }\right),\left(\frac{\left(l_{\max }-l_{d}\right)}{2}\right)\right) \\
l_{s \max } & =l_{\min }-l_{d} \\
g & =l_{s \max }-l_{s \min }
\end{aligned}
$$

If the value of $\mathrm{g}$ in (20) is nonnegative, it means there is the solution to the actuator stroke length of the 6-DOF parallel manipulator under this set of geometric parameters; thus an actuator can be selected to achieve the maximum and minimum leg lengths for the desired total orientation workspace. Otherwise, if the value of $g$ is negative, there is no solution to the actuator stroke length.

\section{Multiobjective Optimization Algorithm}

The Elitist Nondominated Sorting Genetic Algorithm version II (NSGA-II) is one of the most classical and popular multiobjective evolutionary algorithms, which is proposed by Deb [22] et al. In this paper, NSGA-II is employed to solve the multiobjective optimal design problems of 6-DOF parallel manipulator for desired total orientation workspace; the specific process is shown in Figure 6, and the simulated binary crossover (SBX) operator and polynomial mutation operator are applied to the algorithm [11].

4.1. Targets. By the determined volume of the desired total orientation workspace, the larger physical size of the parallel manipulator leads will meet the workspace requirements better. So the physical size of the parallel manipulator needs to be optimized to achieve higher economy and practicability. In general, the maximal leg length [14] or the volume of the manipulator [16] is the target of the physical size of the parallel manipulator.

One of the kinematics and dynamics indexes should be selected as the target to ensure the good performance of the manipulator. The global performance indexes such as the global condition index (GCI) [23], global manipulability index, and global transmission index [17] are calculated over the whole workspace. However, manipulator always works in the desired workspace rather than the whole workspace. Therefore, a performance index is proposed in this paper, which is used for the parallel manipulator within the desired workspace. For example, the desired workspace condition index (DWCI) can be got by

$$
k=\frac{\int_{W} \eta d W}{\int_{W} d W}
$$




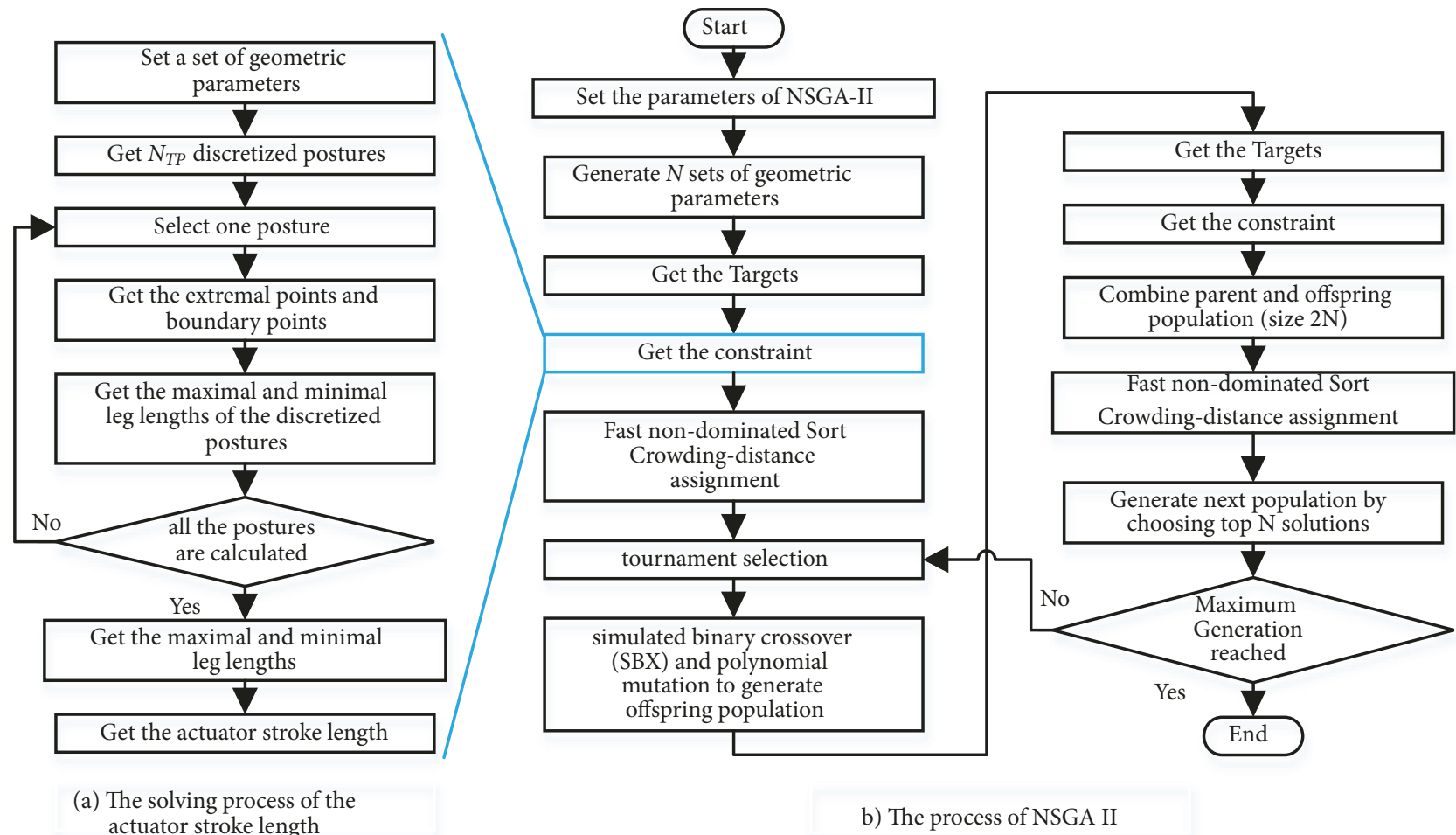

FIGURE 6: The detailed process of NSGA II.

where $W$ denotes the desired workspace and $\eta$ denotes the local conditional index (LCI).

When the Monte Carlo method is used to randomly generate $\mathrm{m}$ poses for the desired workspace, the DWCI can be obtained by (22) [24],

$$
k=\sum_{i=1}^{m} \frac{\eta_{i}}{m}
$$

where $m$ denotes the number of random poses and $\eta_{i}$ denotes the local conditional index of the $i$ th $(i=1, \ldots, \mathrm{m})$ random poses.

The range of $k$ is $(0,1]$, where 0 means the poor dexterity and 1 means the best dexterity. The larger the $k$ is, the more flexible the mechanism is [23].

4.2. Constraint. The actuator stroke length of parallel manipulator for desired total orientation workspace is taken as the constraint. If (20) is nonnegative, the constraint is satisfied. If (20) is negative, the constraint is not satisfied.

Calculate the maximal and minimal leg lengths by the hybrid method proposed in this paper, and then calculate the stroke length. The specific process is shown in Figure 6.

\section{The Example Application}

5.1. The Desired Total Orientation Workspace and Mechanism Configuration. The proposed optimization method is used to optimize the ship motion simulation platform. The ship moves in six DOF, which includes heave (along $z$ axis), sway
TABLE 1: The desired total orientation workspace.

\begin{tabular}{lccc}
\hline & $x$ axis & $y$ axis & $z$ axis \\
\hline Translation Amplitude $(\mathrm{mm})$ & \pm 35 & \pm 35 & \pm 50 \\
Rotation Amplitude $\left({ }^{\circ}\right)$ & \pm 10 & \pm 8 & \pm 5 \\
\hline
\end{tabular}
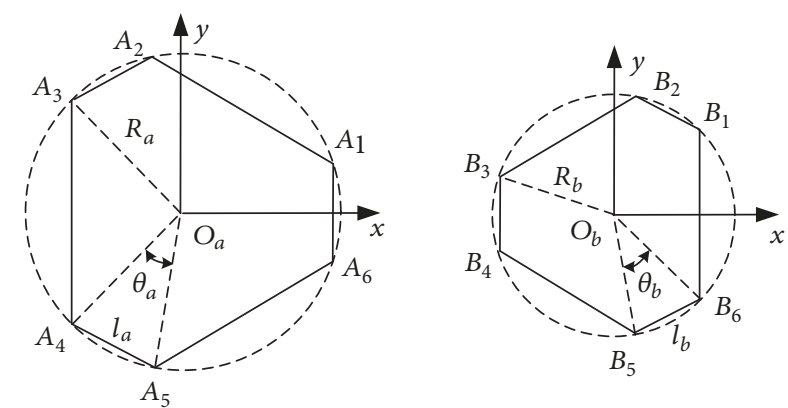

Figure 7: Positions of the joints on the base and moving platform.

(along $y$ axis), surge (along $x$ axis), roll (around $x$ axis), pitch (around $y$ axis), and yaw (around $z$ axis). The amplitudes of roll, pitch, and heave are larger compared to yaw, sway, and surge and have great influence on ship navigation. Therefore, the desired total orientation workspace of ship movement is set as shown in Table 1.

The Stewart parallel manipulator is chosen as the ship motion simulation platform, whose joint position is shown in Figure 7,.where a symmetrical hexagon is constructed by the point $A_{i}$, which is inscribed in a circle with the radius of 
Ra around the point $O_{a} \cdot l_{a}$ denotes the length of the shorter edge of the symmetrical hexagon and $\theta_{a}$ denotes the central angle corresponding to the short side $l_{a}$. Also, a symmetrical hexagon is constructed by the point $B_{i}$, which is inscribed in a circle with the radius of $R_{b}$ around point $O_{b}$. lb denotes the length of the shorter edge of the symmetrical hexagon and $\theta_{b}$ denotes the central angle corresponding to the short sides $l_{b}$.

The central angles $\theta_{a}$ and $\theta_{b}$ can be obtained by (23) and (24) as follows:

$$
\begin{aligned}
& \theta_{a}=2 \arcsin \left(\frac{0.5 l_{a}}{R_{a}}\right) \\
& \theta_{b}=2 \arcsin \left(\frac{0.5 l_{b}}{R_{b}}\right)
\end{aligned}
$$

The vectors $\boldsymbol{a}_{i}$ and $\boldsymbol{b}_{i}$ can be obtained by (25) and (26) as follows:

$$
\begin{aligned}
& \boldsymbol{a}_{i}=\left[\begin{array}{lll}
R_{a} \cos \left(\eta_{i}\right) & R_{a} \sin \left(\eta_{i}\right) & 0
\end{array}\right] \\
& \boldsymbol{b}_{i}=\left[\begin{array}{lll}
R_{b} \cos \left(\varphi_{i}\right) & R_{b} \sin \left(\varphi_{i}\right) & 0
\end{array}\right]
\end{aligned}
$$

where

$$
\begin{aligned}
& \varphi_{i}=\frac{i \pi}{3}-\frac{\theta_{b}}{2}, \quad(i=1,3,5) \\
& \varphi_{i}=\frac{(i-1) \pi}{3}+\frac{\theta_{b}}{2}, \quad(i=2,4,6) \\
& \eta_{i}=\frac{(i-1) \pi}{3}+\frac{\theta_{a}}{2}, \quad(i=1,3,5) \\
& \eta_{i}=\frac{i \pi}{3}-\frac{\theta_{a}}{2}, \quad(i=2,4,6)
\end{aligned}
$$

Therefore, the geometric parameters of Stewart parallel manipulator can be determined by $R_{a}, R_{b}$, and $H$.

5.2. The Cost Function. $R_{a}, R_{b}$, and $H$ are chosen as the design variables of the cost function.

The volume of the Stewart parallel manipulator and the DWCI are chosen as targets of the optimization algorithm. The volume of the Stewart parallel manipulator can be got by (28) as follows:

$$
V=S h=\pi R_{a}^{2} h
$$

The small length of short side leads to the good dexterity of the Stewart parallel manipulator [25]. To ensure the manipulator performs better without the interference of universal (spherical) joints installation, the short side lengths are set as $150 \mathrm{~mm}$. Hydraulic cylinders are chosen as the actuator, and the dead length ld of the hydraulic cylinder is $500 \mathrm{~mm}$. The orientation step length is set as $S_{\alpha}=S_{\beta}=S_{\gamma}=1^{\circ}$.
TABLE 2: The parameters of NSGA-II.

\begin{tabular}{lc}
\hline parameters & values \\
\hline Population size & 50 \\
Number of iterations & 300 \\
Crossover probability & 0.9 \\
Mutation probability & $1 / 3$ \\
Distribution index for SBX & 20 \\
Distribution index for polynomial mutation & 100 \\
\hline
\end{tabular}

The cost function can be expressed as

$$
\begin{aligned}
& \text { Minimize }\left[V\left(R_{a} R_{b} H\right),-k\left(R_{a} R_{b} H\right)\right] \\
& \text { Subject to } 300 \leq R_{a} \leq 700 \mathrm{~mm} \text {; } \\
& 250 \leq R_{b} \leq 700 \mathrm{~mm} ; \\
& 600 \leq H \leq 900 \mathrm{~mm} ; \\
& R_{a} \geq R_{b} ; \\
& l_{a}=l_{b}=170 \mathrm{~mm} ; \\
& S_{\alpha}=S_{\beta}=S_{\gamma}=1^{\circ} \text {; } \\
& l_{d}=500 \mathrm{~mm} ; \\
& \Omega_{\mathrm{t}}=\{(x, y, z, \alpha, \beta, \gamma) \\
& \left.\begin{array}{l}
x_{\min } \leq x \leq x_{\max }, y_{\min } \leq y \leq y_{\max } \\
z_{\min } \leq z \leq z_{\max }, \alpha_{\min } \leq \alpha \leq \alpha_{\max }
\end{array}\right\} \\
& \beta_{\min } \leq \beta \leq \beta_{\max }, \gamma_{\min } \leq \gamma \leq \gamma_{\max } \\
& g=l_{\text {smax }}-l_{\text {smin }} \geq 0 \text {. }
\end{aligned}
$$

5.3. Optimization Results. All the parameters of NSGA-II are presented in Table 2.

The Pareto front, which is the optimization solutions including 50 sets of geometric parameters, is shown in Figure 8. $V$ denotes the volume of the Stewart parallel manipulator, and $k$ denotes the DWCI. The total orientation workspace of the Stewart parallel manipulator of each set of geometric parameters contains the desired total orientation workspace represented by Table 1 . Known from Figure 8 , the large volume of manipulator $V$ leads to the high value of the DWCI $k$. The optimal set of geometric parameters can be selected among the 50 sets of geometric parameters according to the requirements of manipulability, stiffness, or power.

A set of geometric parameters is selected to verify the hybrid method proposed in this paper, as shown in Table 3.

The numerical method and the hybrid method are, respectively, used to obtain the maximal and minimal lengths of 3 legs, and the results are shown in Table 4 . It can be known that the small step length leads to the high precision and long time. The computer runs Windows 7 operating system 
TABLE 3: The set of geometric parameters for verifying the hybrid method.

\begin{tabular}{ccccc}
\hline & Design variables & \multicolumn{2}{c}{ Targets } \\
$R_{a}$ & $R_{b}$ & $H$ & $V$ & $-k$ \\
\hline $522.71 \mathrm{~mm}$ & $322.91 \mathrm{~mm}$ & $853.95 \mathrm{~mm}$ & $7.33 \times 108 \mathrm{~mm}^{3}$ & -0.25917 \\
\hline
\end{tabular}

TABLE 4: The maximal and minimal length of 3 legs.

\begin{tabular}{lccc}
\hline Step length & \multicolumn{2}{c}{ Numerical method } & \multicolumn{2}{c}{ Hybrid method } \\
& $5 \mathrm{~mm}, 1^{\circ}$ & $2 \mathrm{~mm}, 1^{\circ}$ & 1041.0121 \\
Maximum(mm) & 1041.0121 & 1041.0121 & $(35,-35,50,5,8,10)$ \\
pose & $(35,-35,50,5,8,10)$ & $(35,-35,50,5,8,10)$ & 796.3160 \\
Minimum(mm) & 796.3186 & 796.3167 & $(-22.01,-35,-50,-5,-8,-10)$ \\
pose & $(-20,-35,-50,-5,-8,-10)$ & $(-23,-35,-50,-5,-8,-10)$ & 0.33 \\
time/s & 457.47 & 8425.23 & \\
\hline
\end{tabular}

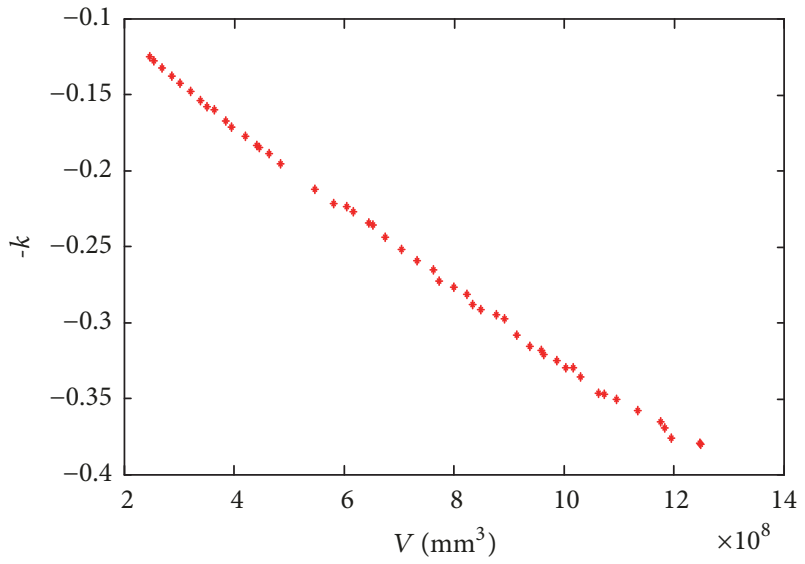

FIGURE 8: The optimization solutions.

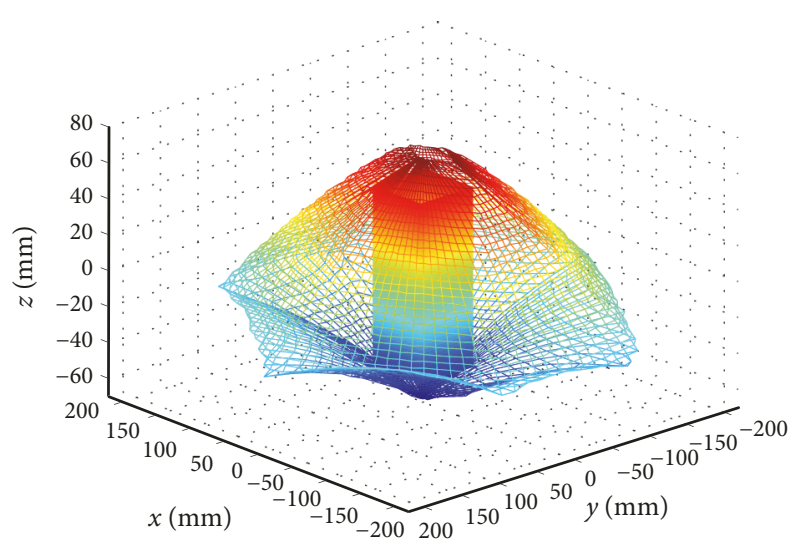

FIGURE 9: The desired total orientation workspace and total orientation workspace.

and has the Intel(R)Core(TM) i7-4770CPU@3.4GHz processor and a $8.00 \mathrm{~GB}$ memory. Compared with the numerical method, the hybrid method is more accurate and efficient, which is suitable for the optimal calculation.

The total orientation workspace defined by the set of geometric parameters of the Stewart parallel manipulator and the desired total orientation workspace shown in Table 1 are shown in Figure 9 [26]. It can be observed that the total orientation workspace contains the desired total orientation workspace.

\section{Conclusions}

A hybrid method for solving the maximal and minimal leg lengths of 6-DOF parallel manipulator with desired total orientation workspace is proposed. The maximal and minimal leg lengths are obtained by the hybrid method, which consists of the numerical method used for orientation variables and algebraic method used for translation variables. The extremal points can be obtained by the hybrid method based on the three-element function without solving six-element function. And compared with the numerical method, it is more accurate and efficient.

The actuator stroke length is obtained to be the constraints, which is calculated by the maximal and minimal length of the legs, which enlarges the optimal range without the predefined maximal and minimal length of the legs.

Aiming at the physical size of the parallel manipulator and the proposed DWCI, the optimization of the geometric parameters of the parallel manipulator is conducted based on the multiobjective optimization algorithm (NSGA-II), which is subject to the actuator stroke length. Stewart platform is set as the example; the geometric parameters of the platform whose workspace contains the desired total orientation workspace are optimized and the hybrid method is proved to be more accurate and efficient compared to the traditional numerical method. This method provides the optimization guidance for engineering designers to design the parallel manipulator for desired total orientation workspace.

\section{Data Availability}

No data were used to support this study.

\section{Conflicts of Interest}

The authors declare that there are no conflicts of interest regarding the publication of this paper. 


\section{Acknowledgments}

This research is supported by the National Natural Science Foundation of China (no. 51875499) and Innovative Research Assistant Support Project of Hebei Province (no. CXZS201801).

\section{References}

[1] Z. Gao, L. Zeng, B. He, T. Luo, and P. Zhang, “Type synthesis of non-holonomic spherical constraint underactuated parallel robotics," Acta Astronautica, vol. 152, pp. 509-520, 2018.

[2] B. He, P. Zhang, and S. Hou, "Accuracy analysis of a spherical 3-DOF parallel underactuated robot wrist," The International Journal of Advanced Manufacturing Technology, vol. 79, no. 1-4, pp. 395-404, 2015.

[3] D. Glozman and M. Shoham, "Novel 6-DOF parallel manipulator with large workspace," Robotica, vol. 27, no. 6, pp. 891-895, 2009.

[4] X. Chen, X.-J. Liu, F. Xie, and T. Sun, "A comparison study on motion/force transmissibility of two typical 3-DOF parallel manipulators: the sprint Z3 and A3 tool heads," International Journal of Advanced Robotic Systems, vol. 11, no. 1, pp. 1-10, 2014.

[5] M. Furqan, M. Suhaib, and N. Ahmad, "Studies on Stewart platform manipulator: a review," Journal of Mechanical Science and Technology, vol. 31, no. 9, pp. 4459-4470, 2017.

[6] Y. Lou, G. Liu, N. Chen, and Z. Li, "Optimal design of parallel manipulators for maximum effective regular workspace," in Proceedings of the 2005 IEEE/RSJ International Conference on Intelligent Robots and Systems, pp. 795-800, Edmonton, Canada, August 2005.

[7] M. Toz and S. Kucuk, "Dexterous workspace optimization of an asymmetric six-degree of freedom Stewart-Gough platform type manipulator," Robotics and Autonomous Systems, vol. 61, no. 12, pp. 1516-1528, 2013.

[8] A. R. Shirazi, M. M. S. Fakhrabadi, and A. Ghanbari, "Optimal design of a 6-DOF parallel manipulator using particle swarm optimization," Advanced Robotics, vol. 26, no. 13, pp. 1419-1441, 2012.

[9] X. J. Liu, J. Wang, and K. Oh, "A new approach to the design of a DELTA robot with a desired workspace," Journal of Intelligent \& Robotic Systems, vol. 39, no. 2, pp. 209-225, 2004.

[10] M. A. Laribi, L. Romdhane, and S. Zeghloul, "Analysis and dimensional synthesis of the DELTA robot for a prescribed workspace," Mechanism and Machine Theory, vol. 42, no. 7, pp. 859-870, 2007.

[11] G. Liu, J. Han, X. Liu, and Z. Qu, "Systematic optimal design procedures for the gough-stewart platform used as motion simulators," Industrial Robot: An International Journal, vol. 40, no. 6, pp. 550-558, 2013.

[12] A. Cirillo, P. Cirillo, G. De Maria, A. Marino, C. Natale, and S. Pirozzi, "Optimal custom design of both symmetric and unsymmetrical hexapod robots for aeronautics applications," Robotics and Computer-Integrated Manufacturing, vol. 44, pp. 1-16, 2017.

[13] J. P. Merlet, Parallel Robots, Springer Science \& Business Media, Mass, USA, 2nd edition, 2006.

[14] J.-S. Zhao, S.-L. Zhang, J.-X. Dong, Z.-J. Feng, and K. Zhou, "Optimizing the kinematic chains for a spatial parallel manipulator via searching the desired dexterous workspace," Robotics and Computer-Integrated Manufacturing, vol. 23, no. 1, pp. 3846, 2007.
[15] J. Fu and F. Gao, "Optimal design of a 3-leg 6-DOF parallel manipulator for a specific workspace," Chinese Journal of Mechanical Engineering, vol. 29, no. 4, pp. 659-668, 2016.

[16] Z. G Yang, M. L. Shao, and D. I. Shin, "Kinematic optimization of parallel manipulators with a desired workspace," Applied Mechanics \& Materials, pp. 752-753-973-979, 2015.

[17] Z. Xie, G. Li, G. Liu, and J. Zhao, "Optimal design of a Stewart platform using the global transmission index under determinate constraint of workspace," Advances in Mechanical Engineering, vol. 9, no. 10, pp. 1-14, 2017.

[18] E. Ottaviano, "A fairly general algorithm to evaluate workspace characteristics of serial and parallel manipulators," Mechanics Based Design of Structures and Machines, vol. 36, no. 1, pp. 1433, 2008.

[19] S. Y. Lu, "Structure of extreme point set of compound function," Academic Forum of Nan Du (Natural Science Edition), vol. 13, no. 1, pp. 26-28, 1993.

[20] C. C. Nguyen, Z.-L. Zhou, S. S. Antrazi, and C. E. Campbell Jr., "Efficient computation of forward kinematics and Jacobian matrix of a Stewart platform-based manipulator," in Proceedings of the IEEE Proceedings of Southeastcon, pp. 869-874, 1991.

[21] D. J. C. Salzmann, Ampelmann: Development of the Access System for Offshore Wind Turbines, BOXPress, 2010.

[22] K. Deb, A. Pratap, S. Agarwal, and T. Meyarivan, "A fast and elitist multiobjective genetic algorithm: NSGA-II," IEEE Transactions on Evolutionary Computation, vol. 6, no. 2, pp. 182-197, 2002.

[23] C. Gosselin and J. Angeles, "A global performance index for the kinematic optimization of robotic manipulators," Journal of Mechanical Design, vol. 113, no. 3, pp. 220-226, 1991.

[24] A. N. Chaudhury and A. Ghosal, "Optimum design of multidegree-of-freedom closed-loop mechanisms and parallel manipulators for a prescribed workspace using Monte Carlo method," Mechanism and Machine Theory, vol. 118, pp. 115-138, 2017.

[25] L. H. Xie, Research on optimal design and dynamic simulation of 6DOF motion platform [Master, thesis], Zhejiang University, China, 2013.

[26] Y. Zhou, J. Niu, Z. Liu, and F. Zhang, "A novel numerical approach for workspace determination of parallel mechanisms," Journal of Mechanical Science and Technology, vol. 31, no. 6, pp. 3005-3015, 2017. 


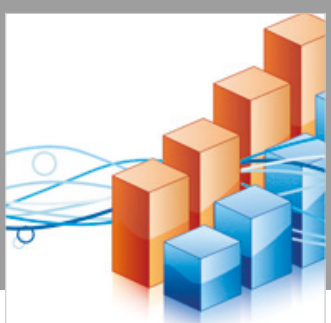

Advances in

Operations Research

\section{-n-m}
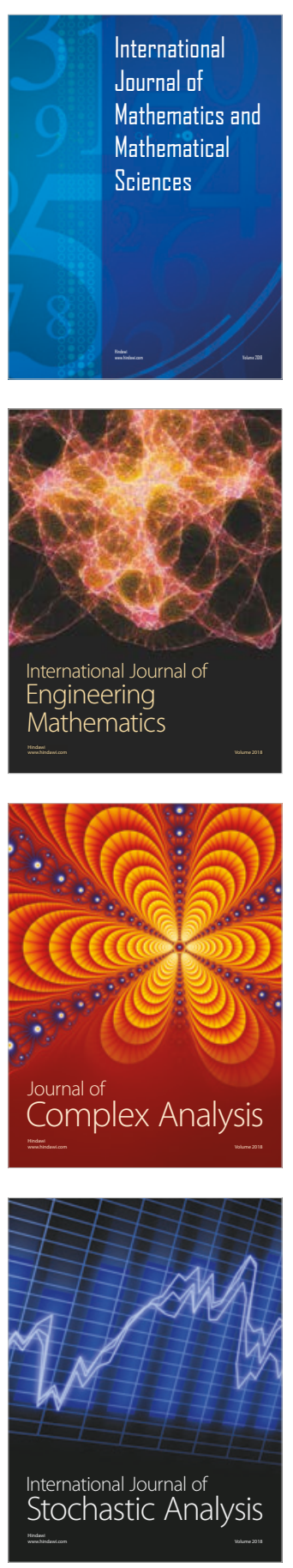
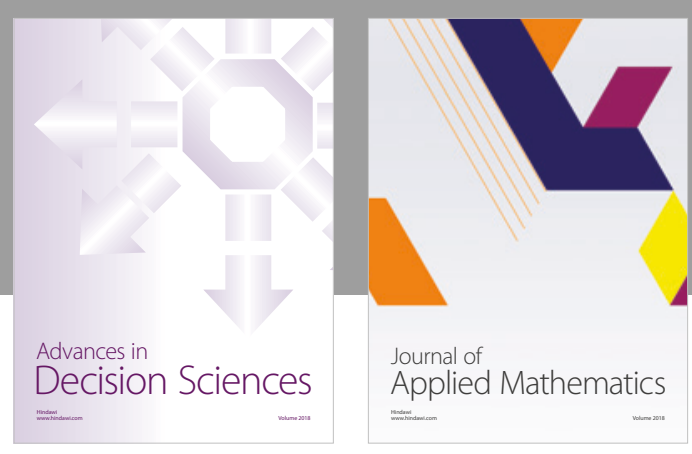

Journal of

Applied Mathematics
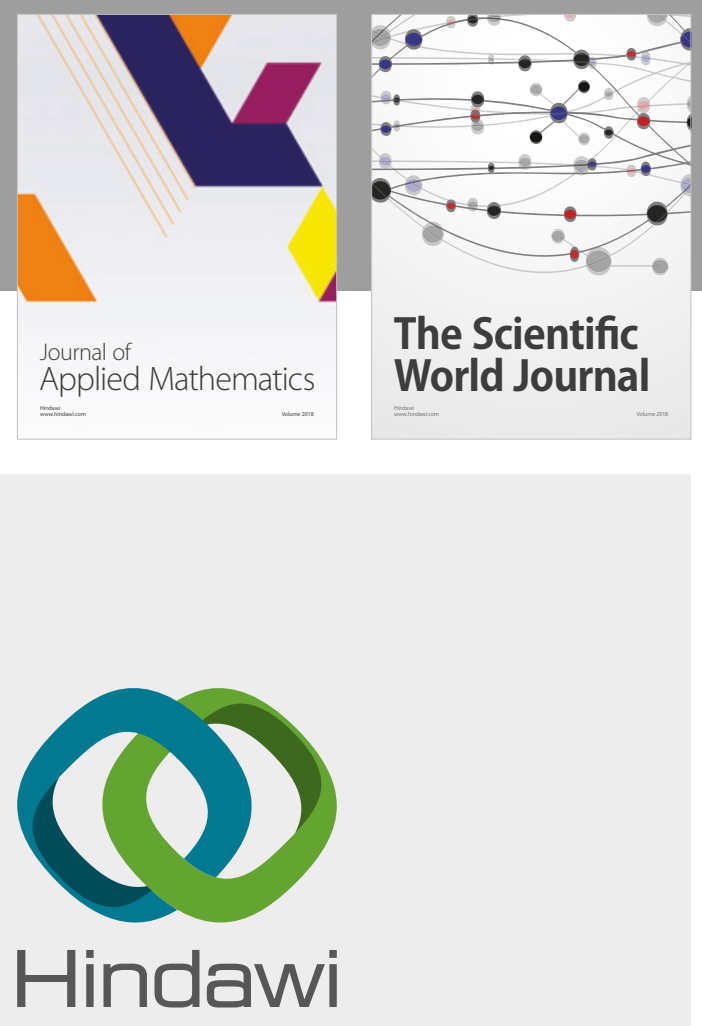

Submit your manuscripts at

www.hindawi.com

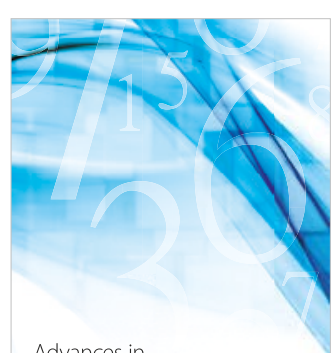

Advances in
Numerical Analysis
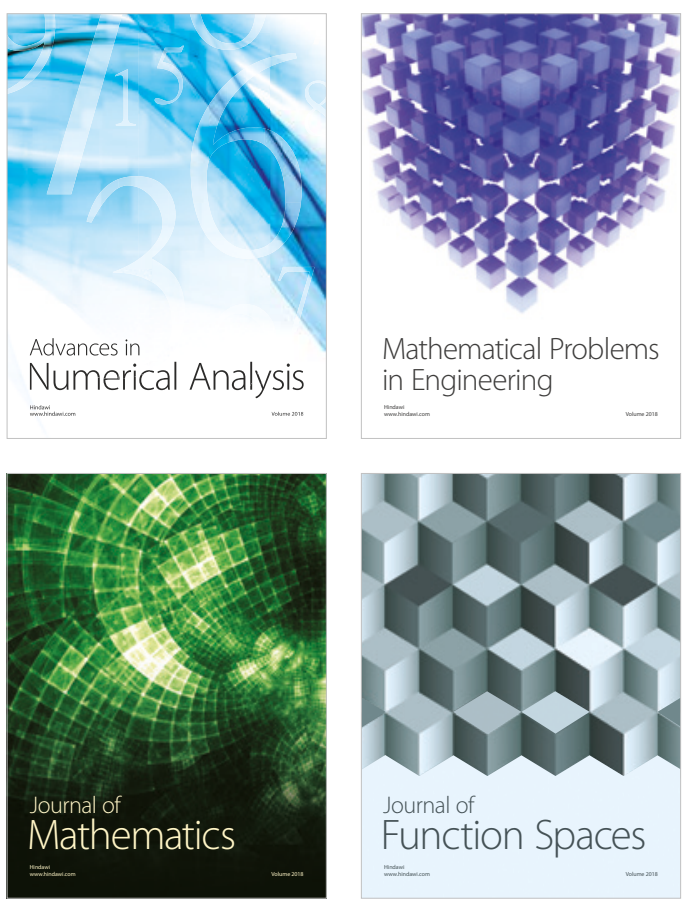

Mathematical Problems in Engineering

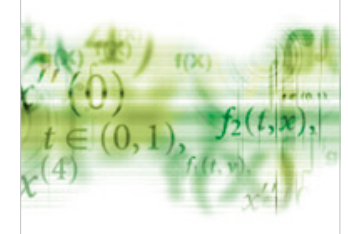

International Journal of

Differential Equations

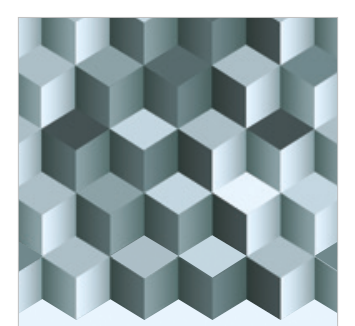

Journal of

Function Spaces
The Scientific

World Journal

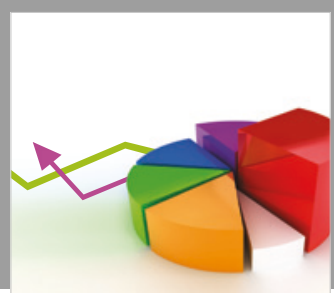

Journal of

Probability and Statistics
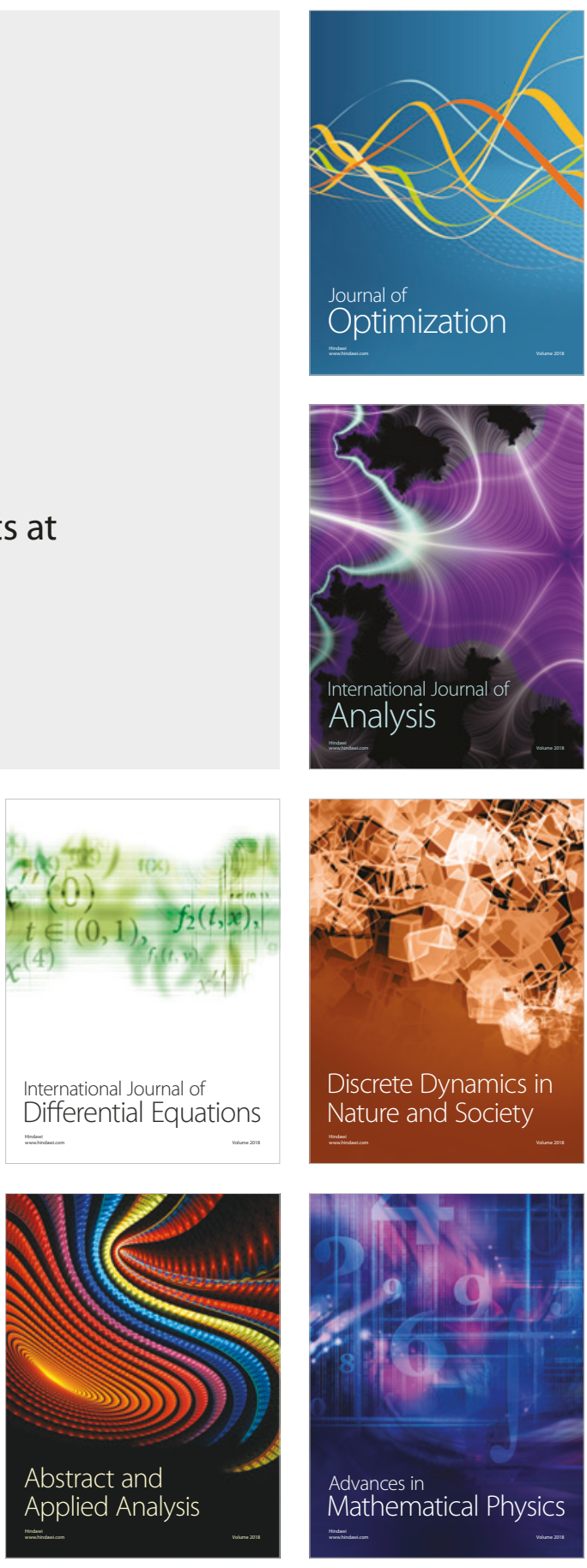